\title{
Injuria miocárdica luego de cirugía no cardíaca
}

\author{
David Torres P. ${ }^{1,2}$, Fernando Botto ${ }^{3}$, Valentina Paz R. ${ }^{1}$ y Javiera Vásquez M. ${ }^{1}$
}

\section{Myocardial injury after non-cardiac surgery}

Major cardiovascular complications in the postoperative period are frequent and the main contributing factor is myocardial injury or infarction (MINS, Myocardial Injury after Noncardiac Surgery) associated with non-cardiac surgery. The 30-day mortality risk in patients with myocardial injury is 4-5 times higher than in patients without this complication. It is estimated that $85 \%$ of patients with MINS/infarction are asymptomatic, hence the only way to detect them is through systematic screening of troponins. Although there are no systematic interventions to prevent this complication, there are treatment and management alternatives, so the active early diagnosis is cost effective and relevant from a clinical point of view.

Key words: perioperative; myocardial infarction; troponin; risk.

\section{Resumen}

Las complicaciones cardiovasculares mayores en el postoperatorio son frecuentes y el principal factor contribuyente es la injuria miocárdica o infarto (MINS, Myocardial Injury after Noncardiac Surgery) asociado a cirugía no cardíaca. El riesgo de mortalidad a 30 días de los pacientes con injuria miocárdica es 4-5 veces mayor que los pacientes que no lo presentan. Se estima que $85 \%$ de los pacientes con MINS no presentan síntomas, por lo que la única manera de detectarlos es mediante screening sistemático de troponinas. Si bien no existen intervenciones de aplicación sistemática que permitan prevenir esta complicación, existen alternativas de tratamiento y manejo, por lo que la pesquisa activa es costo-efectiva y relevante desde el punto de visto clínico.

Palabras clave: infarto; perioperatorio; MINS; troponina; riesgo.

\section{Introducción}

La mortalidad intraoperatoria asociada a anestesia ha disminuido de 1 en 10 mil a 1:100 mil, o más en las últimas décadas. Si bien se considera un evento centinela, su frecuencia lo hace tan improbable que no es un buen desenlace para medir calidad de la atención. Esta disminución de riesgo de más de diez veces en mortalidad intraoperatoria fue un tremendo avance en seguridad, de mayor impacto que otras intervenciones contemporáneas en salud. Requirió un esfuerzo conjunto que incluyó, entre otros, la profesionalización de anestesiólogos, uso de tecnologías (monitores, máquinas, sistemas de seguridad), desarrollo de protocolos y la organización de los lugares de trabajo. Nada de esto se hubiera podido hacer sin antes reconocer que la mortalidad intraoperatoria era un problema real.

Sin embargo, todavía hay un problema que solucionar y es probable que todos los implicados en el cuidado perioperatorio debamos asumir un rol importante. La mortalidad en los 30 días, posteriores a una cirugía mayor no ambulatoria, es mucho más alta que la mortalidad intraoperatoria. Ocurre en el $1-2 \%$ de los pacientes mayores de 45 años con algún factor de riesgo cardiovascular ${ }^{1}$.

El objetivo de esta revisión es presentar las definiciones actuales de daño cardiovascular perioperatorio, estado de la evidencia actual en términos de prevención y manejo, junto a un resumen de la calidad de la evidencia disponible (Tabla 1).

\section{Injuria miocárdica isquémica perioperatoria (infarto perioperatorio)}

El principal factor contribuyente a la mortalidad postoperatoria en cirugía no cardíaca es la injuria miocárdica de origen isquémico, que representa el $34 \%$ de los casos de acuerdo al riesgo poblacional
${ }^{1}$ Anestesiología Universidad de los Andes. Santiago, Chile. ${ }^{2}$ Anestesiología Clínica Santa María. Santiago, Chile.

${ }^{3}$ Departamento de Cardiología e Investigación, Instituto Cardiovascular. Buenos Aires, Argentina.

Recibido el 18 de julio de 2019 y aceptado para publicación el 6 de diciembre de 2019.

Correspondencia a: Dr. David Torres P. dtorresp@gmail.com 
Tabla 1. Calidad de la evidencia y fuerza de la recomendación (GRADE)

\begin{tabular}{|c|c|c|c|}
\hline Sección & Referencia & Calidad de la evidencia & Fuerza de la recomendación \\
\hline MINS y mortalidad & $(2,3,4)$ & Alta & Fuerte a favor \\
\hline Pronóstico MINS vs infarto & $(2,3,6)$ & Alta & Sin efecto \\
\hline Pesquisa mediante screening de troponinas & $(7,8)$ & Alta & Fuerte a favor \\
\hline Betabloqueadores y mortalidad & (9) & Alta & Fuerte en contra \\
\hline Clonidina y MACE & $(10)$ & Alta & Sin efecto \\
\hline Clonidina e hipotensión & $(10)$ & Alta & Débil en contra \\
\hline Aspirina y MACE & (11) & Alta & Sin efecto \\
\hline Aspirina y hemorragia & (11) & Alta & Fuerte en contra \\
\hline \multicolumn{4}{|l|}{ ¿Qué hacer en casos con MINS? } \\
\hline Evaluación cardiológica & $(6,18)$ & Moderada & Débil a favor \\
\hline Prevención secundaria & $(9,18)$ & Baja & Débil a favor \\
\hline \multicolumn{4}{|l|}{ Estudio MANAGE } \\
\hline Dabigatran y mortalidad & $(21)$ & Moderada & Fuerte a favor \\
\hline
\end{tabular}

atribuible (PAR), seguido por la sepsis con el 30\%2. Se ha definido MINS (Myocardial Injury after Noncardiac Surgery) como la elevación de troponinas no explicada por otra causa diferente de la isquémica (tromboembolia pulmonar, sepsis, cardioversión eléctrica, etc.) que puede presentar o no signos y síntomas patognomónicos de isquemia miocárdica aguda, y que engloba al infarto postoperatorio clásico y al silente o inadvertido, y también a cuadros de "isquemia aguda" que elevan la troponina, pero no llegan al grado profundo de necrosis (equivalente a la angina inestable). Esta elevación de troponinas ocurre principalmente en el postoperatorio inmediato, con $87 \%$ en los primeros dos días ${ }^{2}$.

Clásicamente se ha pensado que el riesgo de infarto de miocardio perioperatorio era alrededor de $1 \%$, basado en estudios con baja pesquisa de eventos cardiovasculares ya que, en la era previa a las troponinas, se buscaban con ECG y CPK MB en forma no sistemática. Sin embargo, con la medición sistemática de biomarcadores, aún en sujetos asintomáticos, sabemos que es 8 veces más frecuente ${ }^{2}$.

Del $8 \%$ de los pacientes que presenta MINS, menos de la mitad (42\%) cumple con los criterios de la cuarta definición universal de infarto de miocardio, que es la elevación de biomarcadores sumada a síntomas o signos de daño cardíaco. E1 84\% de MINS no presentó síntomas y el $65 \%$ no tuvo alteraciones isquémicas agudas del ECG.

Esto se explica porque son pacientes graves, con dolor quirúrgico intenso, sometidos a sedación o bajo el efecto de analgésicos potentes, los cuales enmascaran los síntomas e impiden el alerta para realizar ECGs y evaluaciones médicas más profundas. O sea, el $58 \%$ de los casos tiene como única manifestación la elevación aislada de troponinas y no califican como infarto según la cuarta definición universal $^{3,4}$. Por esa razón, ha sido denominada "injuria miocárdica asociada a cirugía no cardíaca" o MINS 5 .

A diferencia de lo que se podría pensar, el infarto no es más grave ni tiene peor pronóstico que MINS. La mortalidad a 30 días de los pacientes con MINS es de $10,4 \%$, comparado con un $8,7 \%$ en los pacientes con infarto ${ }^{6}$. Cuando incluimos la dispersión de los resultados, vemos que no hay diferencias en términos de pronóstico. Donde sí existen diferencias es en el pronóstico de los pacientes con MINS o 
infarto cuando se compara con los pacientes que no lo tuvieron. La mortalidad a 30 días de los pacientes con injuria miocárdica es 10 veces mayor que la de pacientes sin esta complicación $(9,8 \% \text { vs } 11 \%)^{3,6}$. Es por ello que identificar a los pacientes con injuria miocárdica tiene un gran valor pronóstico, y hacerlo precozmente tiene implicancias de monitorización y manejo postoperatorio.

La manera más eficiente de detectar a los casos que cursan MINS es mediante el screening sistemático de troponinas a todos los pacientes de riesgo elevado (Figura 1). El costo de esta estrategia es marginal en relación a los beneficios que supone. Se estima que cada 8 a 10 pacientes de riesgo elevado que se tomen exámenes de troponinas en los primeros dos días postoperatorios se va a detectar un caso de daño miocárdico ${ }^{7}$. El costo de un test de troponinas es aproximadamente de 6 dólares (CLP 3.500), por lo que cada paciente adicional con daño cardíaco que se detecte tendría un valor aproximado de 130 dólares (CLP 90.000). Este método de screening ya se ha utilizado en distintos países, con buen rendimiento, y está incluida en las guías de práctica clínica de riesgo cardiovascular en cirugía no cardíaca más actualizadas ${ }^{7,8}$.

\section{Estudios en morbimortalidad perioperatoria}

En términos de prevención, hasta ahora no se conocen intervenciones que permitan disminuir la probabilidad de presentar un evento, por lo que el riesgo de presentar daño miocárdico depende principalmente de factores no modificables de los pacientes como edad, comorbilidad y tipo de cirugía. Para estudiar intervenciones que permitan disminuir el riesgo postoperatorio se han realizado grandes ensayos clínicos aleatorizados. Por mucho tiempo se pensó que el uso de betabloqueadores en el preoperatorio permitiría disminuir la probabilidad de complicaciones cardiovasculares. El estudio POISE aleatorizó 8.500 pacientes a betabloqueador y placebo y mostró que si bien los pacientes con betabloqueo presentaban menos infarto de miocardio, la

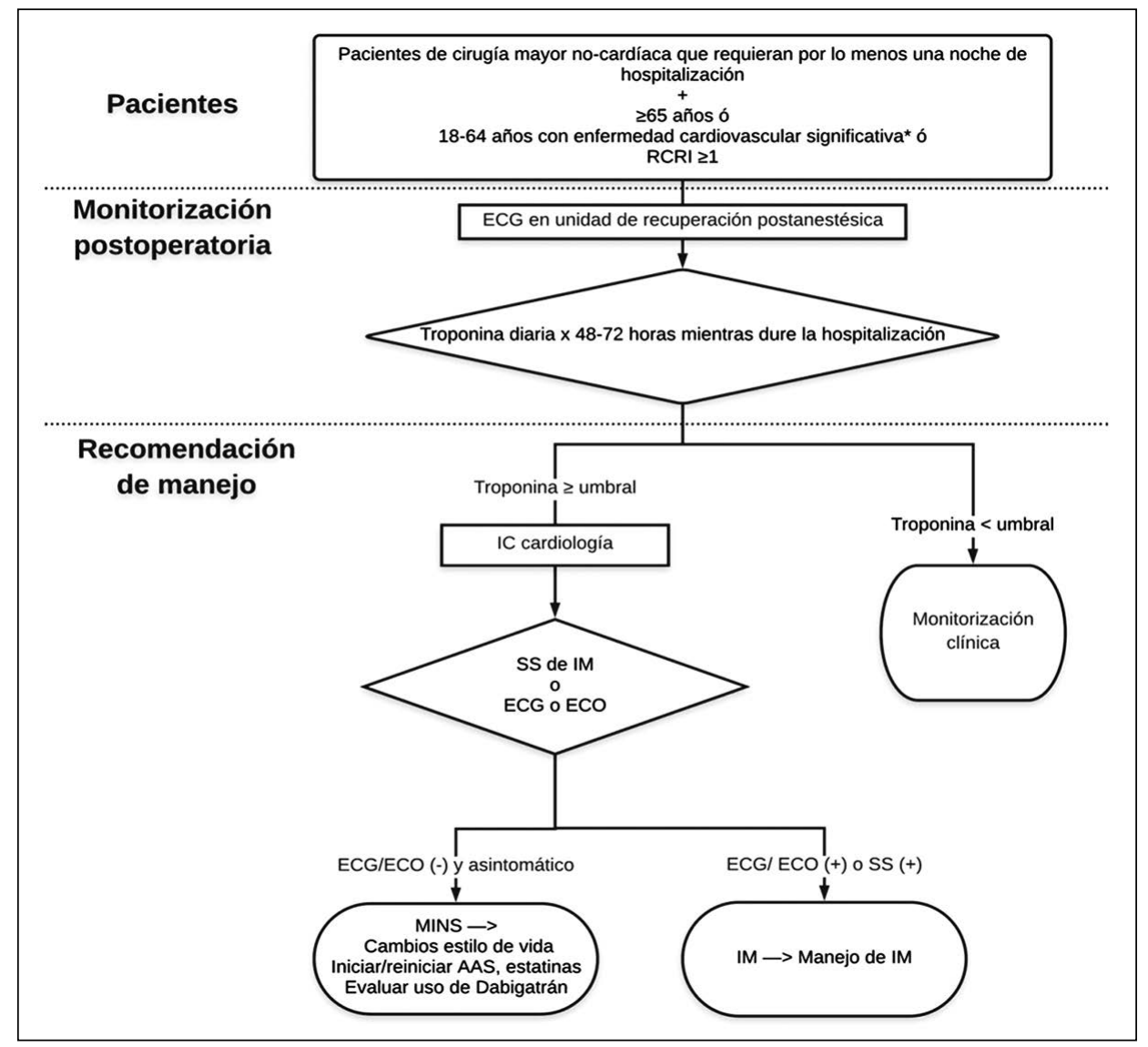

Figura 1. Algoritmo de screening sistemático de troponinas y recomendaciones de manejo en el postoperatorio de pacientes con riesgo cardiovascular elevado. RCRI, índice de riesgo cardíaco revisado; ECG, electrocardiograma; ECO, ecocardiografía cardíaca; IC, interconsulta; SyS, síntomas y/o signos; IM Infarto al miocardio; MINS, injuria miocárdica después de cirugía no cardíaca; AAS, aspirina. *Enfermedad cardiovascular significativa incluye antecedentes de enfermedad coronaria, enfermedad vascular cerebral, enfermedad arterial periférica, insuficiencia cardíaca congestiva, hipertensión pulmonar severa o una anormalidad intracardiaca obstructiva severa (por ejemplo, estenosis aórtica severa, estenosis mitral severa o cardiopatía obstructiva hipertrofia severa): **RCRI (cada uno es un punto): antecedentes de enfermedad coronaria, enfermedad vascular cerebral, enfermedad arterial periférica, insuficiencia cardíaca congestiva, uso preoperatorio de insulina, creatinina preoperatoria $>2 \mathrm{mg} / \mathrm{dL}$ y cirugía de alto riesgo (intraperitoneal, intratorácica o vascular suprainguinal). 
mortalidad era mayor. Esto, debido principalmente a un aumento de la frecuencia de accidentes cerebrovasculares que, cuando se presentaban, además de tener alta letalidad, los sobrevivientes quedaban con secuelas invalidantes ${ }^{9}$.

El estudio POISE-2 fue un ensayo clínico aleatorizado factorial que evaluó dos intervenciones contra placebo. La rama que comparó clonidina y placebo mostró que no existían diferencias en eventos cardiovasculares mayores (muerte, infarto, paro cardíaco no fatal, accidente cerebrovascular (ACV)) y que el uso de clonidina aumenta la probabilidad de desarrollar hipotensión ${ }^{10}$. Por otro lado, la rama que comparó el uso de Aspirina versus placebo observó que la Aspirina no reduce el riesgo de presentar eventos cardiovasculares, y que aumenta la probabilidad de padecer una hemorragia mayor postoperatoria $^{11}$.

El estudio ENIGMA-II sirvió para descartar la impresión que existía que la adición de óxido nitroso a una mezcla de gases aumentaba la probabilidad de complicaciones cardiovasculares cuando se le compara con una mezcla de aire y oxígeno ${ }^{12}$.

En el último tiempo se ha ido acumulando un caudal importante de evidencia que muestra que la hipotensión perioperatoria está asociada a desenlaces cardiovasculares adversos ${ }^{13,14}$. Sin embargo, esta evidencia es observacional, extraída de grandes bases de datos, por lo que los resultados deben ser interpretados con cautela. También existe evidencia observacional y de estudios clínicos aleatorizados pequeños que muestran que el uso de ácido tranexámico en el intraoperatorio permitiría disminuir la hemorragia postoperatoria y consecuentemente, las complicaciones cardiovasculares ${ }^{15,16}$. Hace pocos meses se está realizando en varios países, dentro de los que está incluido Chile, el estudio POISE-3, un ensayo clínico aleatorizado factorial, multicéntrico, que busca evaluar la efectividad del ácido tranexámico comparado con placebo y comparar dos estrategias de manejo de presión arterial en el perioperatorio $^{17}$. Con la incorporación de nuevos centros y la tasa de reclutamiento actual, se estima que los primeros resultados podrían estar disponibles en 3 años.

\section{¿Qué hacer en casos con MINS?}

Pese a que el daño cardíaco perioperatorio tiene una incidencia alta, tiene valor pronóstico y puede detectarse con relativa facilidad, existe la creencia que no es mucho en término de intervenciones lo que se puede hacer para modificar el pronóstico de estos pacientes, por lo que no valdría la pena categorizarlos ni solicitar los exámenes para detectar MINS en el postoperatorio.

En primer lugar, la elevación aislada de troponinas sin otra causa que lo explique, es sinónimo de daño miocárdico perioperatorio con múltiples causas posibles, entre ellas la isquémica. Como tal, debe solicitarse rápidamente un electrocardiograma (ECG) y gatillar una consulta al cardiólogo. Si el ECG no muestra alteraciones isquémicas agudas, aún no podemos descartar infarto o MINS. Considerando la clínica y la magnitud de la elevación de las troponinas, el cardiólogo podrá solicitar un ecocardiograma para evaluar trastornos de la motilidad que puedan señalar infarto o MINS con mayor precisión. Esta diferenciación es relevante, ya que el paciente podría beneficiarse con una angiografía y una eventual intervención coronaria percutánea en casos de compromiso miocárdico extenso ${ }^{5,18}$. De todos modos, la ausencia de trastornos de la motilidad parietal no descarta que sea MINS, dado que la isquemia puede ser transitoria y mejorar, pero tener igualmente pronóstico adverso.

Es probable que los pacientes con MINS se beneficien con el uso de betabloqueadores, Aspirina y estatinas, a partir de datos de estudios observacionales. Los pacientes que ya tomaban estos medicamentos en forma crónica deben reiniciarlos apenas sea seguro hacerlo. Los pacientes que no lo hacían, deben ser evaluados individualmente para ver qué estrategia de prevención secundaria es más beneficiosa ${ }^{19,20}$. Además, es pertinente recomendar cambios al estilo de vida: modificaciones en la dieta, suspensión de tabaco y ejercicio moderado (Figura 1). Estas intervenciones son de bajo costo, seguras $\mathrm{y}$ pueden tener un alto impacto en forma individual ${ }^{5}$.

\section{Estudio MANAGE}

En 2018 se publicaron los estudios del estudio MANAGE, que fue el primer estudio de intervenciones que evaluó la anticoagulación oral en pacientes con MINS/infarto perioperatorio ${ }^{21}$. MANAGE fue un ensayo clínico aleatorizado internacional, factorial (incluyó también omeprazol versus placebo), que estudió 1.754 pacientes aleatorizados en 2 grupos: 877 pacientes recibieron dabigatrán $110 \mathrm{mg}$, 2 veces al día, y otros 877 recibieron placebo. Se evaluó la efectividad en el punto final (PF) primario, que fue un compuesto de complicaciones vasculares mayores, incluida la muerte de origen vascular, en un seguimiento planificado a dos años. El 15\% del grupo placebo presentó el PF primario compuesto, 
comparado con el $11 \%$ del grupo dabigatrán. La reducción de riesgo relativo fue de $27 \%$ y del riesgo absoluto de $4 \%$, que representa un número necesario para tratar de 25 . El desenlace primario de seguridad fue definido como hemorragia masiva o en órganos críticos y ocurrió en 29 pacientes aleatorizados a Dabigatrán y en 31 del grupo placebo ( $3 \%$ vs 4\%, pNS). El desenlace compuesto secundario de seguridad, que agrupaba hemorragia digestiva baja sin relevancia clínica, sangrado menor y dispepsia, mostró una incidencia mayor en el grupo de dabigatrán (15 vs 10\%), consistente con las características del medicamento y la evidencia disponible.

Dentro de las limitaciones del estudio, está el hecho que no se alcanzó a reclutar el tamaño de muestra original; la adherencia al protocolo fue relativamente baja, con un $40 \%$ de pacientes en ambos grupos abandonó el tratamiento antes de completar el tiempo de seguimiento; y el desenlace primario fue modificado en relación al protocolo original para que fuera consistente con otros estudios clínicos ${ }^{21}$. Pese a estas limitaciones, los resultados del estudio MANAGE son coherentes con un importante caudal de evidencia que muestra que los anticoagulantes se asocian a la reducción de eventos isquémicos en pacientes coronarios y su utilidad en tratamientos a largo plazo.

A la luz de estos resultados, surgen nuevas preguntas que debieran dar origen a nuevas líneas de investigación, tal como saber si este efecto se replica con otros anticoagulantes, evaluar el efecto de los antiplaquetarios (clopidogrel o ticagrelor), y cuál es el tiempo óptimo de duración del tratamiento. Estas preguntas nos obligan a ser cuidadosos al extrapolar los resultados de este primer estudio en pacientes con MINS o infarto perioperatorio.

\section{Conclusiones}

En los últimos 30 años se ha logrado reducir la mortalidad en pabellón, haciendo que el periodo in- traoperatorio sea extremadamente seguro. También sabemos que la mortalidad en los primeros 30 días después de una cirugía es muchas veces mayor y que el principal contribuyente en cirugía no cardíaca es la injuria miocárdica perioperatoria de origen isquémico o MINS. En relativamente poco tiempo se ha definido esta complicación de origen cardiovascular que es frecuente ( $8 \%$ en general, y mayor aún en casos de elevado riesgo basal) y que tiene un alto impacto en el pronóstico de los pacientes. También sabemos que la mayoría de los pacientes que la presentan no van a tener síntomas y que la manera más efectiva de diagnosticarlo es el screening sistemático de troponinas. Pese a grandes estudios aleatorizados, no existe hasta el momento alguna intervención que permita disminuir su incidencia en el postoperatorio, pero sí contamos con algún grado de evidencia de cómo enfrentar su tratamiento.

El llamado es a identificar a los pacientes en términos de riesgo cardiovascular, explicar el riesgo y la importancia pronóstica a todo el equipo médico quirúrgico, realizar exámenes postoperatorios a los pacientes de riesgo para poder detectar a los pacientes con MINS y derivar apenas sean detectados para que reciban un manejo multidisciplinario individualizado y evaluar los beneficios de la prevención secundaria (Figura 1).

\section{Responsabilidades éticas}

Protección de personas y animales. Los autores declaran que para esta investigación no se han realizado experimentos en seres humanos ni en animales.

Confidencialidad de los datos. Los autores declaran que en este artículo no aparecen datos de pacientes.

Conflictos de interés: no hay

Financiamiento: Sin apoyo financiero.

\section{Bibliografía}

1. Devereaux PJ, Sessler DI. Cardiac Complications in Patients Undergoing Major Noncardiac Surgery. N Engl J Med. 2015;373:2258-69.

2. Botto F, Alonso-Coello P, Chan MT, Villar JC, Xavier D, Srinathan S, et al. Myocardial injury after noncardiac surgery: a large, international, prospective cohort study establishing diagnostic criteria, characteristics, predictors, and 30-day outcomes. Anesthesiology 2014; 120:564-78.

3. Vascular Events In Noncardiac Surgery Patients Cohort Evaluation Study Investigators, Devereaux PJ, Chan MT, Alonso-Coello P, Walsh M, Berwanger
O, Villar JC, et al. Association between postoperative troponin levels and 30-day mortality among patients undergoing noncardiac surgery. JAMA 2012;307:2295-304.

4. van Waes JA, Nathoe HM, de Graaff JC, Kemperman H, de Borst GJ, Peelen LM, et al. Myocardial injury after noncardiac surgery and its 
association with short-term mortality. Circulation 2013;127:2264-71.

5. Thygesen K, Alpert JS, Jaffe AS, Chaitman BR, Bax JJ, Morrow DA, et al. Fourth Universal Definition of Myocardial Infarction (2018). Circulation 2018;138:e618-e51.

6. Puelacher C, Lurati Buse G, Seeberger D, Sazgary L, Marbot S, Lampart A, et al. Perioperative Myocardial Injury After Noncardiac Surgery: Incidence, Mortality, and Characterization. Circulation 2018;137:1221-32.

7. Biccard BM. Detection and management of perioperative myocardial ischemia. Curr Opin Anaesthesiol. 2014;27:336-43.

8. Duceppe E, Parlow J, MacDonald P, Lyons K, McMullen M, Srinathan S, et al. Canadian Cardiovascular Society Guidelines on Perioperative Cardiac Risk Assessment and Management for Patients Who Undergo Noncardiac Surgery. Can J Cardiol. 2017;33:17-32.

9. Poise Study Group, Devereaux PJ, Yang $\mathrm{H}$, Yusuf S, Guyatt G, Leslie K, et al. Effects of extended-release metoprolol succinate in patients undergoing non-cardiac surgery (POISE trial): a randomised controlled trial. Lancet. 2008;371(9627):1839-47.

10. Devereaux PJ, Sessler DI, Leslie K, Kurz A, Mrkobrada M, Alonso-Coello $\mathrm{P}$, et al. Clonidine in patients undergoing noncardiac surgery. N Engl J Med. 2014;370:1504-13.

11. Devereaux PJ, Mrkobrada M, Sessler DI, Leslie K, Alonso-Coello P, Kurz A, et al.
Aspirin in patients undergoing noncardiac surgery. N Engl J Med. 2014;370:1494503.

12. Myles PS, Leslie K, Chan MT, Forbes A, Peyton PJ, Paech MJ, et al. The safety of addition of nitrous oxide to general anaesthesia in at-risk patients having major non-cardiac surgery (ENIGMA-II): a randomised, single-blind trial. Lancet 2014;384(9952):1446-54.

13. Salmasi V, Maheshwari K, Yang D, Mascha EJ, Singh A, Sessler DI, et al. Relationship between Intraoperative Hypotension, Defined by Either Reduction from Baseline or Absolute Thresholds, and Acute Kidney and Myocardial Injury after Noncardiac Surgery: A Retrospective Cohort Analysis. Anesthesiology 2017;126:47-65.

14. Sessler DI, Meyhoff CS, Zimmerman NM, Mao G, Leslie K, Vásquez SM, et al. Period-dependent Associations between Hypotension during and for Four Days after Noncardiac Surgery and a Composite of Myocardial Infarction and Death: A Substudy of the POISE-2 Trial. Anesthesiology 2018;128:317-27.

15. Crash- trial collaborators, Shakur H, Roberts I, Bautista R, Caballero J, Coats $\mathrm{T}$, Dewan Y, et al. Effects of tranexamic acid on death, vascular occlusive events, and blood transfusion in trauma patients with significant haemorrhage (CRASH-2): a randomised, placebo-controlled trial. Lancet 2010;376(9734):23-32.

16. Woman Trial Collaborators. Effect of early tranexamic acid administration on mortality, hysterectomy, and other morbidities in women with postpartum haemorrhage (WOMAN): an international, randomised, doubleblind, placebo-controlled trial. Lancet 2017;389(10084):2105-16.

17. Devereaux PJ, Population Health Research Institute. PeriOperative ISchemic Evaluation-3 Trial (POISE-3) clinicaltrials.gov2018 [Available from: https://clinicaltrials.gov/ct2/show/ NCT03505723.

18. Devereaux PJ. Suboptimal Outcome of Myocardial Infarction After Noncardiac Surgery: Physicians Can and Should Do More. Circulation. 2018;137:2340-3.

19. Devereaux PJ, Xavier D, Pogue J, Guyatt G, Sigamani A, Garutti I, et al. Characteristics and short-term prognosis of perioperative myocardial infarction in patients undergoing noncardiac surgery: a cohort study. Ann Intern Med. 2011;154:523-8.

20. Foucrier A, Rodseth R, Aissaoui M, Ibanes C, Goarin JP, Landais P, et al. The long-term impact of early cardiovascular therapy intensification for postoperative troponin elevation after major vascular surgery. Anesth Analg. 2014;119:1053-63.

21. Devereaux PJ, Duceppe E, Guyatt G, Tandon V, Rodseth R, Biccard BM, et al. Dabigatran in patients with myocardial injury after non-cardiac surgery (MANAGE): an international, randomised, placebo-controlled trial. Lancet 2018;391(10137):2325-34. 